\title{
Low Cost Arduino Based Voice Controlled Pick and Drop Service with Movable Robotic Arm
}

\author{
Shaikh Khaled Mostaque and Bishal Karmakar
}

\begin{abstract}
This paper presents the designing and implementation of low cost Arduino based Robotic Arm with ability of picking and placing the object as instructed by human voice. The robot also contains a feature of detection of object by itself through sonar technique before proceeding voice commands. Such a system can be helpful in enabling users to do works in industry or mining applications without risking their lives and bringing more perfection. The user just has to own a Smartphone which is present in almost every ones hand and the robot. The voice instruction can be transmitted to robot via Bluetooth interface with Android Apps. Arduino Mega works as the brain that controls the arm and makes decision before grabbing any object.
\end{abstract}

Index Terms-Arduino Mega, HC-05 Bluetooth Module, Micro Servo, Sonar Sensor, Voice Command.

\section{INTRODUCTION}

Controlling with voice and doing anything with less human touch is a trending topic in the $21^{\text {st }}$ century. With the flow of time, it is putting an impact in our daily lives. With less human effort, commands are provided to others with relative dexterity through utterance of words. When the first computer was exposed in front of the mankind, communication using voice to a machine was only realized in science fictions. However with formidable breakthroughs in the field of science, we are at the sheer drop of truly using our voice to command and control electronic devices [1].

The main attraction of this kind of automation is reducing human efforts, time, life risk, error due to human negligence and bringing perfection. Using wireless technology in the area of robotics it is possible to move robots freely and work on difficult areas which are either inaccessible or dangerous for human presence. For instance, NASA's mission to Mars, the Spirit and Opportunity Drone and implementing highly explicit medical treatments can be taken into consideration .However, it is realized that there are several factors(e.g. deterioration of signal quality due to propagation effects like attenuation and dispersion apart from electromagnetic interference) that restrain the rapid progress towards full scale implementation of wireless technology [2].The term Smartphone is an important part of our life now a day due to the development of modern technology. Applications of Smartphone are being developed based on android system. Among all available mobile operating systems Android OS

Published on November 21, 2016.

Shaikh Khaled Mostaque is a faculty member of Dept. of Electrical and Electronic Engineering, Varendra University, Rajshahi, Bangladesh. (misha@vu.edu.bd, skm.misha@gmail.com).

Bishal Karmakar is from Department of Electrical and Electronic Engineering, Varendra University, Rajshahi, Bangladesh (bishal.karmakar95@gmail.com). has gained significant and immense popularity due to its open architecture. This platform has opened new dimensions for technical innovation and exploration [3].

In this paper, we introduce a robotic arm controlled by voice through natural language processing in Android OS. The arm is placed on a moving vehicle. Through different commands the robot is able to move in any direction and stop. The arm uses four micro servo motors for the pick and place operation. It consists of an arm assembly with a jaw. One servo allows the robotic hand to rotate $180^{\circ}$ and another one allows the hand to move in only up and down direction. The third one is attached with the up and down structure that gives an advantage to move the arm in forward and backward direction. The fourth servo is used for the opening and closing of the jaw. For the simplicity of operation and minimization of cost, locally available components and scraps were used to make the body of the moving vehicle and the arm assembly. For the controlling of motors, driver ICs and Arduino Mega 2560 microcontroller board was used [4]. This microcontroller contains 15 PWM channels among which 8 are needed to drive servo and the dc motors. The controlling signal was given serially from an Android OS based smart phone, which is interfaced with the Arduino board via Bluetooth module through android apps. Thus, the voice command enables the robot to go to a remote place, pick and grab a sample object and drop it in a desired place. A two way check of detection of object before grabbing through voice command was also integrated with our robot using sonar sensing technology. Such a feature helps the robot to take decision of grabbing object only if it is present nearby.

\section{LiteratURE REVIEW}

Shyam.R.Nair and Shalini.R.Nair in their paper adapted a remote operation system for a robotic arm in [5]. One of the two disadvantages of their work is their robotic hand can't move from one place to another place. The second one is whenever it receives command it starts grabbing whether there is an object or not. R. Aswinbalaji and A.Arunraja presented a similar type of work with their robot in [6].

A group of researchers in [7] worked on three way controlled android OS based robotic vehicle. The robot was only able to move forward, backward, left right or stop as per chosen application via Android OS platform. However, the platform was inbuilt touch screen sensors in Smartphone rather than using speech recognition. Researchers in [8] discussed a hardware and software co-design of a Robot Arm Controller with 5 motors but with using large FPGA.

The proposed system is an improved design of robot that can move forward and backward with the robotic hand through wheels. Whenever it gets a command for grabbing 
through voice, it checks the presence of object with the help of sonar sensor.

\section{SYSTEM DESIGN}

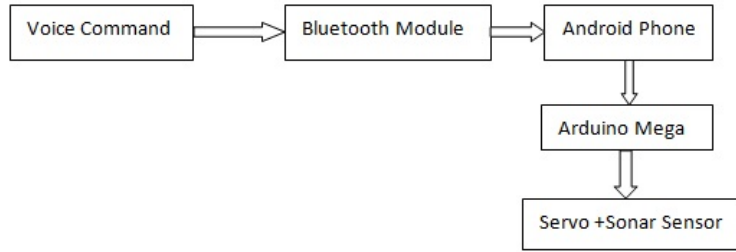

Fig. 1. Block Diagram of the System

The Voice-operated Android based robotic hand with self detection uses an Android based Bluetooth enabled phone for its application, Arduino Mega as the microcontroller, servos as the hand and sonar sensor for self detection.

\section{A. Arduino Mega}

The Arduino Mega 2560 is a programming platform which traditionally called microcontroller board based on the ATmega2560 [9]. It consists 54 digital input/output pins among them 15 can be used as PWM (Pulse Width Modulation) outputs. There are 16 analog inputs and 4 are used as hardware serial ports. It also contains a $16 \mathrm{MHz}$ crystal oscillator for oscillation, a USB connection for burning the code, a power jack for giving power to the board, an ICSP header, and a reset button. The Mega2560 does not used FTDI USB-to-serial driver chip. This feature separates the Mega from other Arduino models. Instead of using FTDI USB-to-serial driver chip, it characterizes the Atmega8U2 programmed as a USB-to-serial converter [4].

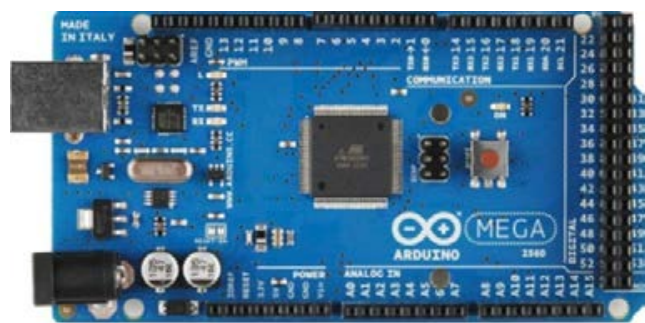

Fig 2: Arduino Mega 2560

\section{B. SG90 Micro Servo}

The SG90 Micro Servo motors are small and lightweight (only 9g) with high output power. They can rotate 180 degrees (90 degree in both direction), and works just like the standard servos but smaller in size. Any arduino code for servo, hardware or library can be called for specific performance. It comes with a 3 horns (arms) and hardware. Its stall torque is $1.8 \mathrm{kgf.cm}$ and operating speed is $0.1 \mathrm{~s} / 60$ degree [10].

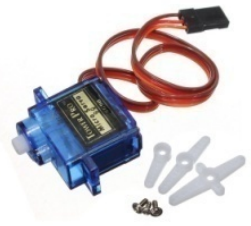

Fig.3. SG90 Micro Servo

\section{HC-SR-04 Sonar Sensor}

The HC-SR-04 ultrasonic sensor operates with the same principal that bat and dolphin's uses to locate an object. Ultrasonic sensor uses sound propagation to calculate distance between sensor and object. It has some excellent features such as zero-contact range detection with high accuracy and reliable data reading. It has calmness operating range of $2 \mathrm{~cm}$ to $400 \mathrm{~cm}$ or 1 ” to 13 feet. Sunlight or black material (such as Sharp rangefinders) can't resist its operation. Ultrasonic transmitter and receiver complete the HC-SR-04 Ultrasonic sensor module [11].

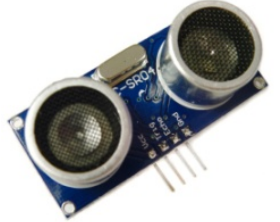

Fig.4. HC-SR 04 Sonar sensor

\section{L293D DC Motor Driver}

L293D is a motor driver IC which follows dual H-bridge principal. It takes a low-current control signal as input and provides a higher-current output signal which is used to drive the dc motors. Two dc motors can be driven at the same time in both forward and reverse direction under normal operating condition. Input logics are provided at Pin $2 \& 7$ and $10 \& 15$ to control the motor operation. The corresponding motor will stop when input logic is 00 or 11 . Clockwise and anticlockwise rotations are performed by Logic 01 and Logic 10 in a relative manner [12].

\section{E. HC-05 Bluetooth Module}

HC-05module is fully qualified with Bluetooth V2.0 with EDR(Enhanced Data Rate) and 3Mbps modulation with complete $2.4 \mathrm{GHz}$ radio transceiver and baseband. The module is created for see-through wireless serial connection setup. It works with CSR Bluecore 04-external single chip system with CMOS technology and Adaptive Frequency Hopping feature [13].

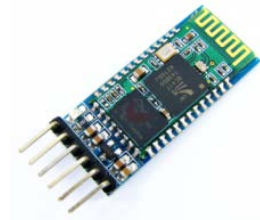

Fig.5. HC-05 Bluetooth Module

\section{F. Android meets robot}

Android meets Robot is a platform for internal voice recognition system in Android Smartphone's. It can be used to communicate with Arduino through voice commands using HC-05 module. It helps to pair Android device with the module and sends voice commands as strings. Thus any microcontrollers that can handle strings can easily use it for processing voice commands [14]. 


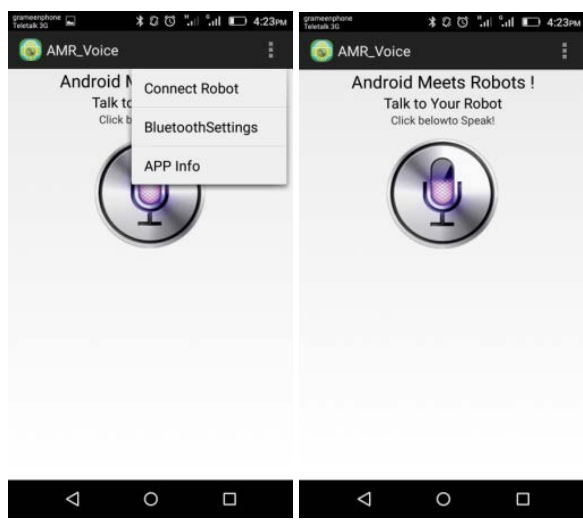

Fig.6. Interface for the Voice Control Application

\section{VOICE COMMANDS RECOGNITION}

A. Commands for movement through wheel

The following commands do the tasks when they are communicated with the robot via Bluetooth module. The system is speaker independent. If the robot does not understand the user commands it gives the user feedback via text in android software.

Forward: This command will make robot to go forward.

Backward: This command will make robot to come backward

Left: With this command, the right wheel will rotate forward and left wheel will rotate in backward direction.

Right: It will do the opposite i.e. the left wheel will rotate forward and the right wheel will be reversed.

\section{B. Commands for robotic arm}

The arm consists of four sections each containing a micro servo motor. The first one is the base which can rotate from left to right and vice versa. The second one moves the arm in forward and backward direction. The third one moves in up and down direction to pick or drop the object to a desired place. The last one is used for jaw movement. The following commands are applicable for above tasks. The robot executes commands only if the object is detected by robot itself through sonar sensor.

SL-Servo1 will turn the whole hand part left.

SR-servo1 will turn the whole hand part right.

SF- servo2 will start rotating to a prefixed angle and the whole hand will go forward.

SB- servo2 will again rotate to a prefixed angle and the whole hand will come backward.

SU- servo3 will make the upper portion of the hand to go upward direction.

SD-servo4 will make the upper portion of the hand to move in downward direction.

OPEN and CLOSE commands are used for grabbing the object.

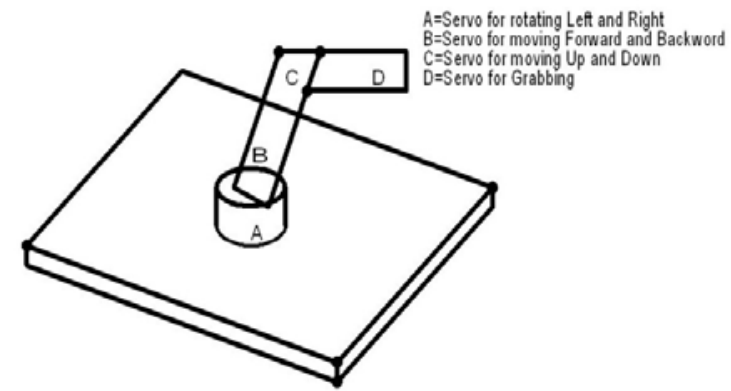

Fig.7. 2D diagram of the robotic hand

\section{FLOW CHART}

A. Movement of robot through wheel

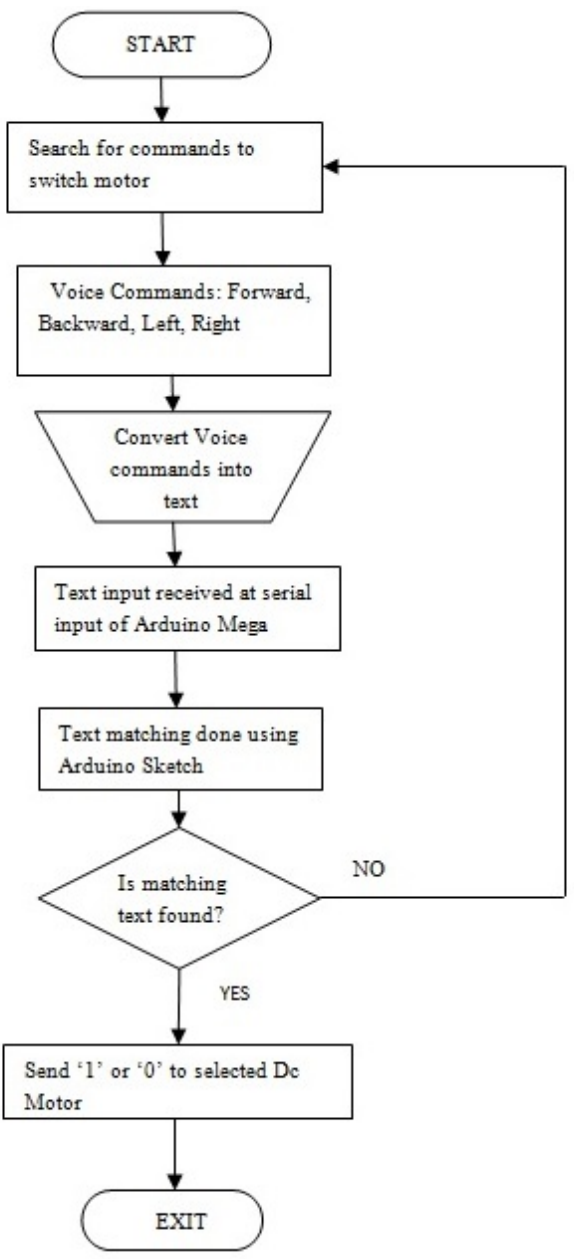

Fig.8. Flow chart for movement through wheel 


\section{B. Movement and function of robotic arm}

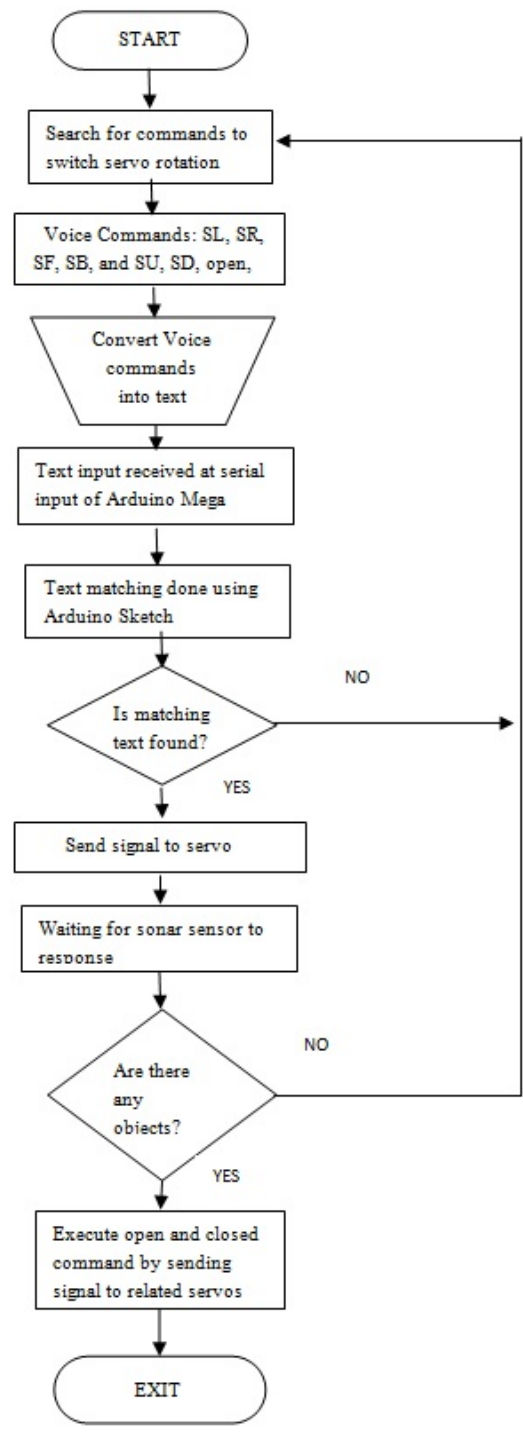

Fig.9. Flow chart for robotic arm

\section{CONCLUSION}

In this paper, we have implemented a mobile robotic arm which can be moved to a desired place to pick or drop any object. The whole process is instructed by voice commands via android apps. We have proposed a two way algorithm to perform the above tasks. The use of sonar technology has provided the feature of self detection before executing the grab command. Further, the robot provides a text to the user if the voice is not properly recognized. The system has been developed with Arduino board and Micro Servo motors. The use of Smartphone and android platform has made the system speaker independent. To minimize the overall cost, we have considered locally available components and scratches to build the structure of vehicle as well as assembly of jaw and the arm. The future selection of hand characteristics and specification will depend upon tradeoffs between shape and weight of object, dexterity and control methods.

Our proposed model can be useful in different applications in superstores, laboratories, medical technologies, industries, mining, rescue missions and in workplaces that are dull or dangerous for human.

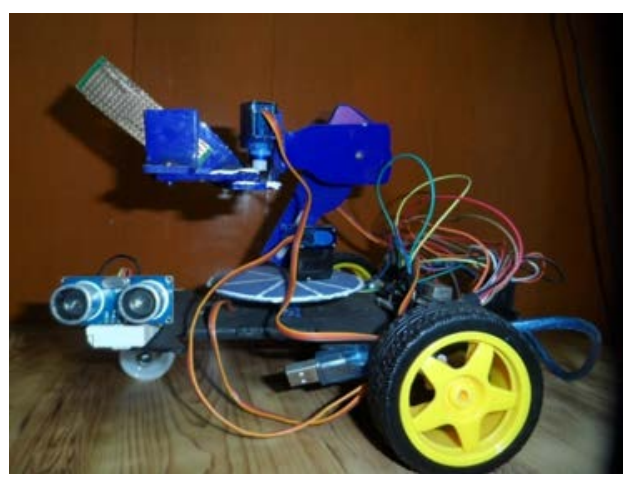

Fig.10. One dimensional View of the Robot

\section{REFERENCES}

[1] S.Sen, S.Chakrabarty, R.Toshniwal, A.Bhaumik, "Design of an Intelligent Voice Controlled Home Automation System”. International Journal of Computer Applications, Vol: 121(15), 2015.

[2] M. Muhammed, S.Z.M. Muzi, S.R.Zakaria, M.Z.M.Jenu,” MR999-E Wireless Robotic Arm”, Intl. Conference on ICT for the Muslim World, Malaysia, 2006.

Available:http://eprints.uthm.edu.my/2726/1/Paper_ID_62.pdf

[3] S.Gobel, R.Jubeh, S.L Raesch, A.Zundorf, "Using the Android Platform to control Robots”, Germany.

Available:http://www.innoc.at/fileadmin/user_upload/_temp_/RiE/Pr oceedings/65.pdf

[4] Available: https://www.arduino.cc/en/Main/ArduinoBoardMega2560

[5] S.R.Nair, S.R.Nair, "Design of a Voice Controlled Robotic Arm for Picking and Placing an Object”, IOSR Journal of Engineering , Vol 2(4), pp 670-673, April 2012.

[6] R.Aswinbalaji, A.Arunraja "Wireless Voice Controlled Robotics Arm”, ISSN: pp 0976-1353 Vol. 12(4), 2015.

[7] A. Dey, A. Pal, S. Nandi, L. Roy, "Three Way Controlled Android Smartphone based Robotic Vehicle via Bluetooth”, International Journal of Advanced Research in Computer and Communication Engineering, Vol. 4(9), 2015.

[8] U.D. Meshram, R. Harkare, "Fpga based five axis robot arm controller”, International Journal of Electronics Engineering, Vol 2(1), pp 209-211, 2010.

[9] Atmel ATMEGA 2560 Datasheet, Available: www.atmel.com

[10] SG90 Datasheet, Available: http://www.micropik.com/PDF/SG90Servo.pdf

[11] HC-SR04 Ultrasonic Sensor User's Manual, Cytron Technologies. Available: https://docs.google.com/document/d/1YyZnNhMYy7rwhAgyL_pfa39RsB-x2qR4vP8saG73rE/edit

[12] L293D Datasheet, Available: http://www.engineersgarage.com/electronic-components/l293dmotor-driver-ic

[13] HC-05 Module Datasheet, Available: http://www.robotshop.com/media/files/pdf/rb-ite-12bluetooth_hc05.pdf.

[14] Available: http://www.instructables.com/id/Voice-Activated-ArduinoBluetooth-Android/ 


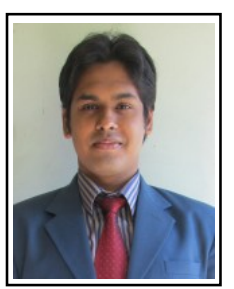

Shaikh Khaled Mostaque was born in Rajshahi, Bangladesh on 26 December, 1989. He has completed his M.Sc. and B.Sc. (Honours) in Applied

Physics and Electronic Engineering from University of Rajshahi, Bangladesh in 2011 and 2010 respectively.

and Biomedical Engineering.

is currently working as a Lecturer in Varendra University, Rajshahi, Bangladesh. His research interests include Human Robot Interaction, Photonics

Mr. Mostaque is an active member of American Corner and World Literature Centre, Bangladesh. He got NST Fellowship for 5G Communications based M.Sc. Thesis work from Govt. of Ministry of Science and Technology, Bangladesh and successfully passed IT Passport Examination which is offered by Information Technology Professionals Examination Council (ITPEC) and adopted by Information Technology Promotional Agency, Japan.

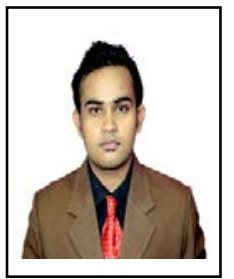

Bishal karmakar comes from Bangladesh .His date of birth is 30.09.1995. He is currently doing his final year of B.Sc. in Electrical and Electronic Engineering from Varendra University, Rajshahi, Bangladesh.

He is been working on robotics for two years. He is mentoring the students of E-club on Basic Robotics, Arduino Programming and Embedded Design in Varendra University, Rajshahi, Bangladesh.

Mr.Karmakar have earned the $1^{\text {st }}$ runner up position in Robodroid Championship, 2016 arranged by IEEE in RUET, among all the teams from different universities o Bangladesh. 